\title{
Uso de biomarcadores para el diagnóstico de enfermedad articular en el caballo
}

\author{
Use of biomarkers for the diagnosis of joint disease in horses
}

\author{
H Chávez ${ }^{\mathrm{a}}$, O Araya ${ }^{\mathrm{b}}$, H Folch $^{\mathrm{c}}$, G Morán $^{\mathrm{d} *}$

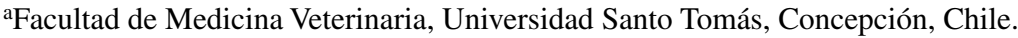 \\ bInstituto de Ciencias Clínicas Veterinarias, Facultad de Ciencias Veterinarias, Universidad Austral de Chile, Valdivia, Chile. \\ 'Instituto de Inmunología, Facultad de Medicina, Universidad Austral de Chile, Valdivia, Chile.

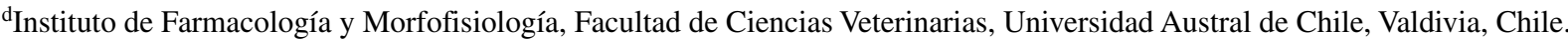

\section{SUMMARY}

\begin{abstract}
Lameness associated to joint disease (JD) in athlete equines is an important cause of economic losses. Intensive training produces biomechanical overload of the synovial joints with a consequent activation of metalloproteinases, which have been associated to the pathogenesis of equine JD. This disease is characterized by the degradation of the extracellular matrix (ECM) of the articular cartilage causing the release of structural components of the cartilage matrix to the joint space and producing changes in the synovial fluid concentration of these ECM components, termed biomarkers. The changes in the concentration of some of these proteins in the synovial fluid might be used to evaluate both the onset and progression of equine JD. The measurement of the levels of aggrecan and collagen type II in the synovial fluid using specific monoclonal antibodies is an example of this approach. This review presents an update on biomarkers used for equine JD.
\end{abstract}

Palabras clave: enfermedad articular, biomarcador molecular, líquido sinovial, caballo.

Key words: joint disease, biomarkers, synovial fluid, horse.

\section{INTRODUCCIÓN}

Las claudicaciones asociadas con enfermedad articular (EA) son las causas más comunes en la limitación del rendimiento físico de los caballos de deporte, el cual constituye una importante causa de pérdida económica en la industria equina (Preston y col 2008). El dolor articular responsable de la claudicación está relacionado con la liberación al espacio articular de neurotransmisores, mediadores de la inflamación, con consecuente disminución del umbral nociceptivo en respuesta a la agresión articular (Bertone y col 2001, de Grauw y col 2006ª). En el caballo de deporte, la carga biomecánica sobre las articulaciones durante el ejercicio intenso y repetitivo ha sido reconocida como un componente central en la etiopatogénesis de EA (McIlwraith 1996). La EA del caballo incluye la osteoartritis (OA), osteocondritis (OC) y el fragmento osteocondral (FO), artropatías caracterizadas por una reacción sinovial con progresiva degradación del cartílago articular y hueso subcondral (Kidd y col 2001). El trauma articular que se produce durante el ejercicio provoca microdaño difuso y remodelación del hueso subcondral, acumulación de estrés óseo, muerte celular (necrosis y apoptosis) y esclerosis. Este fenómeno promueve la degradación del cartílago articular por

Aceptado: 03.06.2009.

\# Programa MECESUP AUS 0061.

* Casilla 567, Valdivia, Chile; gmoran@uach.cl incongruencia con la base ósea subcondral (Cruz y Hurtig 2008). Adicionalmente, modificaciones metabólicas en el cartílago articular y membrana sinovial inflamada, asociadas o no al trauma articular, activan el sistema enzimático de las enzimas metaloproteinasas (MMPs), desencadenando la degradación del cartílago articular (Clutterbuck y col 2008, Sutton y col 2009). Este proceso produce fibrilación del cartílago articular por pérdida de moléculas estructurales de la matriz extracelular (MEC), provocando inicialmente disfunción articular con eventual desarrollo de la EA (Huber y col 2000, D'Lima y col 2001).

El diagnóstico de EA en el caballo se puede lograr a través de la exploración clínica, análisis del líquido sinovial (LS) y estudio de imágenes radiográficas, ecográficas o también mediante gamagrafía, tomografía computarizada y resonancia magnética (Steel 2008); además, mediante procedimientos invasivos como la artroscopia (McIlwraith 2002). Sin embargo, hasta ahora imágenes articulares con evidencias de lesiones no han sido logradas sino hasta cuando un importante y permanente daño ha ocurrido en el tejido articular (Billinghurst 2003).

En los últimos años han emergido métodos no invasivos para el estudio de EA en pacientes humanos y caballos, denominados biomarcadores. Un biomarcador (BM) se define como cualquier indicador molecular, directo o indirecto, de recambio anormal del tejido articular, que en propiedad corresponde a un componente normal o a un subproducto de los procesos metabólicos del tejido articular (cuadro 1) (Garnero y col 2000, Billinghurst 2003, van Weeren y 
Cuadro 1. Biomarcadores del metabolismo articular osteocondral utilizados para el diagnóstico de enfermedad articular en equinos. Biomarkers of joint metabolism used in the diagnosis of joint disease in horses.

\begin{tabular}{|c|c|c|}
\hline \multicolumn{3}{|c|}{ Biomarcadores del metabolismo articular osteocondral } \\
\hline $\begin{array}{l}\text { Biomarcadores directos } \\
\text { del metabolismo del cartílago }\end{array}$ & $\begin{array}{l}\text { Biomarcadores del proceso anabólico } \\
\text { Biomarcadores del proceso catabólico }\end{array}$ & Agrecán colágeno tipo II \\
\hline $\begin{array}{l}\text { Biomarcadores indirectos } \\
\text { del metabolismo del cartílago }\end{array}$ & $\begin{array}{l}\text { Deshidrogenasa láctica (LDH) } \\
\text { Metaloproteinasas (MMPs) } \\
\text { Eicosanoides } \\
\text { Proteínas de fase aguda } \\
\text { Citoquinas } \\
\text { Ácido hialurónico }\end{array}$ & \\
\hline Biomarcadores del & $\begin{array}{l}\text { Biomarcadores del proceso } \\
\text { anabólico }\end{array}$ & $\begin{array}{l}\text { Fosfatasa alcalina (FA) } \\
\text { Osteocalcina (Oc) } \\
\text { Propéptidos de colágeno tipo II }\end{array}$ \\
\hline óseo & $\begin{array}{l}\text { Biomarcadores del proceso } \\
\text { catabólico }\end{array}$ & $\begin{array}{l}\text { Piridinodina (Pyr)-Deoxipiridinolidina (D-Pyr) } \\
\text { Telepéptidos de colágeno tipo II } \\
\text { Sialoproteína ósea }\end{array}$ \\
\hline $\begin{array}{l}\text { Biomarcadores misceláneos } \\
\text { de enfermedad articular }\end{array}$ & $\begin{array}{l}\text { Mieloperooxidasa (MPO) } \\
\text { Proteína sensible al ácido retinoico der }\end{array}$ & a del cartílago (CD-RAP/MIA) \\
\hline
\end{tabular}

Firth 2008). La degradación de la MEC del cartílago articular ocurre tempranamente en la EA, liberando al LS componentes moleculares posibles de cuantificar (Laverty y col 2000, Arai y col 2008, Frisbie y col 2008). Así como ha avanzado el entendimiento de la EA equina, un desafío permanente ha sido identificar BMs de daño articular más específicos y sensibles para interpretar correctamente sus modificaciones. Alcanzar este objetivo puede facilitar el diagnóstico precoz de alteraciones metabólicas del tejido articular, antes que se registren imágenes de su degradación, ofreciendo con ello una anticipada información de utilidad en la clínica equina.

\section{CONSIDERACIONES ANATOMOFUNCIONALES DEL CARTÍLAGO ARTICULAR}

El tejido articular que compone y sostiene la superficie de la articulación sinovial se denomina tejido osteocondral. Histológicamente, el cartílago articular ocupa el estrato superficial del tejido osteocondral otorgando parte de los límites a la cavidad sinovial (Muller-Gerbl 1998). El cartílago articular está constituido por una pequeña población de células mesenquimales llamadas condrocitos, las cuales alcanzan al menos el $1 \%$ del volumen del cartílago. Los condrocitos están dispuestos en una extensa MEC compuesta fundamentalmente de agua, colágeno, proteoglicanos y glicoproteínas (Huber y col 2000). Todos los componentes de la MEC del cartílago articular son sintetizados por los condrocitos, los que elaboran una variedad de MMPs y sus inhibidores tisulares, encargados del recambio físiológico y patológico de la MEC (Poole 1993, Poole y col 2001). En este proceso, los condrocitos regulan su actividad metabólica a través de señales biomecánicas, eléctricas y fisicoquímicas emitidas desde la MEC (Blain 2007). Así, la carga fisiológica cíclica es una señal mecánica central en el metabolismo normal de los condrocitos (Kim y col 1994, Todhunter 1996).

El principal colágeno del cartílago articular maduro es el colágeno tipo II, que alcanza un $42-45 \%$ del peso seco (Eyre 2002). El colágeno tipo II es una proteína fibrilar formada de una molécula precursora llamada tropocolágeno, que sintetiza y secreta los condrocitos como procolágeno tipo II (Garnero y col 2000). El colágeno forma una malla tridimensional particularmente distribuida en cada zona del cartílago articular, dotando a este tejido de particulares propiedades biomecánicas de resistencia a las cargas de tensión (Huber y col 2000).

Interrelacionados mecánica y químicamente con la malla tridimensional de colágeno tipo II, se encuentran los proteoglicanos (PG). El agrecán es el mayor PG del cartílago articular maduro (Rosenberg 1993, Roughley 2006). El monómero de agrecán es una macromolécula compleja constituida por una proteína central (PC) que se fija por enlaces covalentes a cadenas laterales de glicoaminoglicanos (GAGs). En el cartílago articular maduro los principales GAGs unidos a la PC son los disacáridos condroitín-6-sulfato (CS) y keratan sulfato (KS) respectivamente, que proveen al cartílago articular de resistencia a las fuerzas de compresión (Dijkgraaf y col 1995). En el cartílago articular normal los PGs están en un constante recambio, regulado por la actividad anabólica y catabólica de los condrocitos (Hardingham y Fosang 1995). Además, el agrecán experimenta modificaciones metabólicas durante el desarrollo del animal (Platt y col 1998, Caterson y col 2000). Áreas específicas y fracciones de la degradación en el recambio de los PGs constituyen la base molecular de marcadores de anabolismo y catabolismo del cartílago articular (Garnero y col 2000). 


\section{BIOMARCADORES DEL METABOLISMO} ARTICULAR OSTEOCONDRAL

El empleo de BMs en el estudio de EA equina ha sido reportado en numerosos estudios sobre OA (Frisbie 2002, Frisbie y col 2003, Ley y col 2007, Frisbie y col 2008), OC (Billinghurst y col 2004, Donabédian y col 2008) y FO (Frisbie y col 1999, Arai y col 2008, Trumble y col 2009). El examen cuantitativo de BMs de EA se puede realizar en muestras de suero, LS y orina, por medio de técnicas de análisis bioquímico-colorimétricas e inmunológicas. Estas últimas, de mayor sensibilidad, utilizan anticuerpos poli y monoclonales, logrando respuestas inmunoespecíficas contra diversos fragmentos y epítopes de metabolito articular osteocondral (Frisbie y col 1999, Donabédian y col 2008, Fietz y col 2008 ${ }^{\mathrm{a}}$ ).

\section{BIOMARCADORES DIRECTOS DEL METABOLISMO DE CARTÍLAGO}

\section{BIOMARCADORES DE PROCESOS ANABÓLICOS DEL CARTÍLAGO}

Agrecán. El desarrollo de anticuerpos monoclonales ha permitido reconocer en el cartílago articular diversos epítopes nativos en la región del agrecán rica en CS. Un anticuerpo que ha recibido atención es el condroitín sulfato 846 (CS-846), el cual reconoce al epítope del mismo nombre presente en el agrecán de reciente síntesis. Este epítope se expresa normalmente en el cartílago articular fetal y escasamente en el cartílago adulto sano (Rizkalla y col 1992). Estudios de Frisbie y col (1999) en caballos con FO (de varios grados) en las articulaciones radiocarpiana e intercarpiana demostraron aumento significativo de los niveles sinoviales y urinarios del epítope CS-846, respecto a los valores en articulaciones normales, sugiriendo su utilidad en el diagnóstico de FO equina. En cambio, el epítope CS-846 disminuye significativamente en el LS de potrillos con lesiones OC en la articulación tarsocrural respecto de los animales sanos (Laverty y col 2000). El epítope CS-846 en LS equino puede diferenciar cambios en el cartílago articular atribuibles al ejercicio de aquellos animales con OA (Frisbie y col 2003). Por otro lado, Frisbie y col (2008) encontraron aumentos significativos del epítope CS-846 sérico y en LS en caballos en ejercicio con OA con respecto de aquellos animales sometidos a un esfuerzo, no siendo modificado con la administración de antiinflamatorios no esteroidales (Fradette y col 2007).

Colágeno tipo II. La síntesis de colágeno tipo II está directamente relacionada con la liberación de los telopéptidos aminopropéptido (N-telopéptido) y carboxipropéptido (C-telopéptido) de procolágeno tipo II (CPII) (Poole 1993, Poole y col 2001). Diversos anticuerpos han sido desarrollados para reconocer y cuantificar el CPII en EA humana. Posteriormente, estudios en caballos con FO han demostrado aumento significativo en las concentraciones sinoviales (Ray y col 1996) y séricas (Frisbie y col 1999, Frisbie y col 2005) de CPII. Del mismo modo, caballos jóvenes con OC tarsocrural presentaron aumento significativo de CPII en LS, respecto de las articulaciones sanas (Laverty y col 2000). Esta elevación del CPII sérico tuvo una relación temporal significativa (durante el primer año de vida) con lesiones de OC en la articulación femorotibial y tibiotarsal diagnosticadas radiográficamente (Billinghurst y col 2004). Por otra parte, algunos autores sugieren que los niveles de CPII en LS radiocarpiano pueden variar por la presencia de antiinflamatorios esteroidales, observándose un descenso de esta proteína con metilprednisolona (Robion y col 2001) y un aumento de los niveles con triamcinolona (Céleste y col 2005). Por otro lado, un programa de entrenamiento intenso en caballos normales incrementó significativamente los niveles de CPII en el LS carpiano. Esas articulaciones en caballos osteoartríticos sometidos a ejercicio experimentaron un incremento adicional de CPII respecto de los animales sanos (Frisbie y col 2008).

\section{BIOMARCADORES DE PROCESOS CATABÓLICOS DEL CARTÍLAGO}

Glicosaminoglicanos. La cuantificación de los GAGs totales fue el primer método que se utilizó para evaluar la degradación del cartílago articular en caballos con OA. Sin embargo, las estimaciones de GAGs en EA equina han revelado resultados contradictorios, entre ellas el origen que tienen los GAGs en el LS. Se ha comprobado que el cartílago articular es la principal fuente de GAGs en el LS; no obstante, otros tejidos como la membrana sinovial y ligamentos pueden liberar GAGs al espacio articular (Ray y col 1996). Además, la concentración de GAGs en LS de caballos sanos presenta diferencias entre distintas articulaciones y áreas articulares, en función del nivel de la carga mecánica que reciben (Fuller y col 1996, Brama y col 2002). Estudios de Alwan y col (1991) en caballos con $\mathrm{OA}, \mathrm{OC}$, artritis traumática y artritis séptica comprobaron concentraciones significativamente altas de GAGs en el LS respecto a los valores hallados en los animales controles (Frisbie y col 2003, Bolam y col 2006). En contraste con estos hallazgos, investigaciones de van den Boom (2005) en caballos con OA metacarpofalángica no encontraron una correlación entre los valores de GAGs en LS y la gravedad de las lesiones en el cartílago articular, situación que le restó validez al BM. Resultados similares reveló un estudio en caballos mestizos con OA metacarpofalángica, en el que no se demostraron diferencias estadísticamente significativas entre distintos grados de la enfermedad con respecto a los animales controles (Adarmes y col 2006). Del mismo modo, niveles de GAGs en LS de caballos con OC disecante tarsocrural no mostraron diferencias entre articulaciones enfermas y normales. Sin embargo, una investigación reciente en caballos de carrera con FO de la articulación carpiana mostraron niveles de GAGs en LS significativamente menores que en las articulaciones controles (Adarmes y col 2008). Por último, Frisbie y 
col (2008) mostraron concentraciones elevadas de GAGs séricos en caballos con $\mathrm{OA}$ experimental sometidos a ejercicio comparados con caballos controles. Esto sugiere que este BM podría ser de utilidad para diferenciar estas dos condiciones en particular.

Keratán sulfato. El keratán sulfato (KS) ha sido evaluado en numerosos estudios como indicador de recambio del cartílago articular equino. Investigaciones iniciales en caballos en los que se empleó un anticuerpo monoclonal (5-D-4) demostraron aumentos significativos de KS en LS de articulaciones con OA, comparado con articulaciones normales (Alwan y col 1991). Además, Todhunter y col (1993) en artritis equina experimental le asignaron al KS sinovial valor como BM de procesos articulares inflamatorios agudos. Sin embargo, trabajos que evaluaron el KS sérico y sinovial en caballos normales y con FO de la articulación radiocarpiana e intercarpiana mostraron escasa utilidad diagnóstica del KS en el estudio de esa artropatía (Frisbie y col 1999). Por otra parte, en caballos jóvenes con OC tarsocrural se han observado concentraciones significativamente bajas de epítopes de KS en LS (Laverty y col 2000), al igual que en caballos adultos con EA aséptica (Misumi y col 2002). Sin embargo, Fuller y col (2001) demostraron en caballos con OA carpiana y metacarpofalángica un nivel significativamente bajo de KS (5-D-4) en LS de articulaciones clínicamente alteradas respecto de las normales. Estas variaciones del KS en el LS podrían deberse a cambios metabólicos del cartílago articular bajo condiciones patológicas. Del mismo modo, Bertone y col (2005) mostraron en el cartílago articular equino normal concentraciones de KS significativamente menores al contenido en el cartílago osteoartrítico y en el cartílago con FO reparado. Por último, algunos autores sugieren que el incremento fisiológico del KS sérico en caballos sanos en entrenamiento de carrera puede reflejar el efecto de la carga articular del ejercicio sobre la actividad metabólica del cartílago (Okumura y col 2002).

Fragmentos de colágeno tipo II. La presencia de fragmentos de la degradación del colágeno tipo II se ha investigado en EA equina, mediante el desarrollo de anticuerpos que identifican epítopes ocultos luego de la hidrólisis enzimática del colágeno, epítopes $\mathrm{C} 1-2 \mathrm{C}$ y C2C, respectivamente (Poole 1993). Un anticuerpo específico de la degradación del colágeno tipo II en la especie equina (234CEQ) fue desarrollado por Billinghurst y col (2001). Estudios en potrillos con OC demostraron una correlación positiva, entre las concentraciones séricas de C2C y 234CEQ, respectivamente, con los registros radiográficos de la lesión en el cartílago (Billinghurst y col 2004). Los epítopes 234CEQ y C2C aumentan significativamente en el LS de caballos osteoartríticos en ejercicio en comparación con aquellos animales sin lesión sometidos a un esfuerzo (Frisbie y col 2008). En cambio, en caballos de carrera con lesiones osteocondrales de las articulaciones del carpo y del menudillo se observó un incremento significativo de C2C en LS, en comparación con los niveles observados en articulaciones de caballos sanos en reposo y en ejercicio (Trumble y col 2009).

Proteína oligomérica de la matriz del cartílago. La proteína oligomérica de la matriz del cartílago (COMP) es una glicoproteína no colágena, pentamérica, estructuralmente relacionada con la trombospodina aislada desde el condrocito. Evidencias sugieren que la COMP une los procolágenos y colágenos I y II (interacción $\mathrm{Zn}^{2+}$ dependiente), teniendo una importante función en la organización de la MEC y fibrinogénesis del colágeno (Rosenberg y col 1998). Inicialmente, la COMP fue estudiada en el caballo como BM del recambio del cartílago articular. Sin embargo, esta proteína también se expresa en otros tejidos como tendón, membrana sinovial y ligamentos (Smith y Heinegard 2000). Las concentraciones de COMP en el cartílago articular equino parecen estar relacionadas con varios factores, entre ellos la raza y el tipo de ejercicio (Skiöldebrand y col 2001, Skiöldebrand y col 2005)). Del mismo modo, mediante la utilización de un anticuerpo monoclonal murino (12C4) contra COMP humana en caballos de carrera con OA, OC, y FO se observaron niveles séricos de COMP significativamente elevados respecto de los controles sanos (Misumi y col 2002). El desarrollo de un anticuerpo monoclonal contra la COMP de cartílago articular equino (mAb 14G4) ha permitido estudios más específicos. Así, empleando este anticuerpo en caballos de carrera con OA, Arai y col (2005) mostraron concentraciones sinoviales de COMP significativamente altas en comparación con los caballos del grupo control. Por otro lado, el análisis de COMP en orina puede tener valor como BM predictivo de OA (Misumi y col 2006), así como de FO en caballos de carrera (Arai y col 2008), cambiando significativamente en función del ejercicio y del tipo de articulación afectada (Helal y col 2007).

Proteína central. El principal epítope de la proteína central (PC) se produce en la región N-terminal del agrecán en respuesta a varias artropatías humanas, estimulando la producción de anticuerpos contra esa proteína en LS (Karopoulos y col 1996). Sin embargo, esta proteína es de escaso valor diagnóstico en caballos con EA.

\section{BIOMARCADORES INDIRECTOS DEL METABOLISMO ARTICULAR}

Los BMs indirectos del metabolismo articular son moléculas sintetizadas en el tejido articular o que acceden a éste, afectando de manera importante el metabolismo de esos tejidos (Sutton y col 2009).

Deshidrogenasa láctica. La enzima deshidrogenasa láctica (DHL) cataliza la reducción del piruvato por NADH para formar lactato, siendo un indicador de la actividad 
metabólica del tejido articular (Mathews y col 2002). En el cartílago articular, la actividad DHL se ha detectado sólo en el citosol de los condrocitos (Hozokawa y col 1988). Una investigación de Yancik y col (1987) en EA equina demostró que la actividad DHL en el LS puede ser de utilidad en el estudio de esta enfermedad. Sin embargo, estos mismos autores comprobaron que la actividad DHL es similar en caballos con EA degenerativa, sinovitis idiopática u OC. Al respecto, un estudio sobre caracterización del hueso subcondral equino demostró que la actividad DHL fue significativamente inferior en lesiones de OC menos agresivas (van de Lest y col 1999). Por tanto, este BM no discrimina el tipo de lesiones que pueden originarse en la EA.

Metaloproteinasas. Las MMPs son un grupo de endopeptidasas $\mathrm{Zn}^{2+} \mathrm{y} \mathrm{Ca}^{2+}$ dependientes, con un papel central en el recambio fisiológico y patológico de la MEC del cartílago articular. El desequilibrio en la actividad de estas enzimas y sus inhibidores tisulares puede llevar a una progresiva degradación de la MEC como ocurre en la EA (Bramono y col 2004). Las MMPs tienen diversas fuentes celulares, principalmente sinoviocitos y condrocitos, pero también neutrófilos y monocitos circulantes, desde donde se liberan como pro-MMPs (Clegg y col 1997ª). La activación de las MMPs es fundamental en la patogénesis de la EA (Clegg y col 1999). Clegg y col $\left(1997^{b}\right)$ han reportado niveles elevados de actividad enzimática de isoformas bioactivas de MMP-2 y MMP-9 en el LS equino con EA aséptica y séptica. Asimismo, Trumble y col (2001) demostraron que la actividad de la isoforma pro-MMP-2 fue significativamente superior en el LS de caballos con EA traumática y OC; no obstante, la actividad de la pro-MMP-9 sólo estuvo elevada en animales con artritis séptica. Por otro lado, en articulaciones equinas normales la actividad enzimática de la MMP-1 en LS puede disminuir con la edad y no se afecta por acción del ejercicio (Brama y col 2004). En tanto, se ha observado que la actividad enzimática de las MMPs totales en equinos se puede incrementar significativamente en el LS metacarpofalángico, correlacionado positivamente con el daño del cartílago articular (van den Boom y col 2005). Asimismo, un estudio de Kidd y col (2007) sugirió que la razón pro-MMP9:pro-MMP2 sumado al recuento globular blanco podría ser un buen indicador de pronóstico en caballos con artritis séptica. Finalmente, Fietz y col $\left(2008^{\mathrm{a}}\right)$ reportaron elevaciones significativas de la actividad de MMP-2 y MMP-9 en la EA séptica con respecto de las articulaciones normales.

Eicosanoides. La prostaglandina $\mathrm{E} 2\left(\mathrm{PGE}_{2}\right)$ es un reconocido mediador de la inflamación y ha sido estudiada como BM en EA equina. Estudios de Gibson y col (1996) demostraron que sólo la $\mathrm{PGE}_{2}$ incrementa significativamente en el LS de caballos con FO y EA degenerativa respecto a las articulaciones normales. Lo propio establecieron Bertone y col (2001) en un estudio en EA equina, sugiriendo que una única medición de $\mathrm{PGE}_{2}$ en el LS podría tener valor diagnóstico predictivo de algunas artropatías. Por otro lado, asociando valores de $\mathrm{PGE}_{2}$ y otros $\mathrm{BM}$ se lograría discriminar entre lesiones del cartílago articular inducidas por diferentes causas. De esto último, relacionando niveles de $\mathrm{PGE}_{2}$ y epítope CS-846 del LS equino se podrían segregar lesiones del cartílago inducidas por el ejercicio de aquellas promovidas por OA (Frisbie y col 2003). El daño articular asociado con $\mathrm{OC}$ en el caballo muestra concentraciones de $\mathrm{PGE}_{2}$ en LS significativamente superiores a las registradas en articulaciones control. Lo mismo fue observado en caballos en ejercicio con OA, en los que se observó que los niveles de $\mathrm{PGE}_{2}$ en el LS fueron significativamente superiores respecto de los valores en ejemplares normales en ejercicio (Frisbie y col 2008).

Proteínas de fase aguda. Existe escasa documentación sobre la respuesta inflamatoria de fase aguda en artropatías equinas no infecciosas y su eventual utilización como BM. Un estudio en caballos sobre la respuesta sistémica de fase aguda en artritis no infecciosa del carpo demostró diferencias significativas entre los niveles séricos de amiloide A sérico (AAS), haptoglobina, fibrinógeno y globulina- $\alpha_{2}$. Las concentraciones de AAS mostraron la más rápida elevación después de inducir artritis experimental (Hultén y col 2002). Del mismo modo, se han observado niveles séricos y sinoviales elevados de AAS en caballos con artritis infecciosa y tenovaginitis séptica (Jacobsen y col 2006). Sin embargo, faltan más estudios para observar la real utilidad clínica de este BM en caballos.

Citoquinas. Las citoquinas son importantes reguladores de la respuesta inmune. Tienen un rol central en el desarrollo de lesiones articulares (Goldring y Goldring 2004). Bertone y col (2001) asignaron a la IL-6, TNF- $\alpha$ e IL-1 $\beta$ valor diagnóstico como marcadores de EA. IL-6 y el IL-1 $\beta$ han sido asociadas con la resorción ósea patológica observada en la lesión quística subcondral del caballo (von Rechenberg y col 2001). Por otra parte, investigaciones de Semevolos y col (2001) en muestras de cartílago equino con OC demostraron concentraciones significativamente elevadas del TGF- $\beta_{1}$ respecto de esos valores en muestras de cartílago control (Fortier y col 2005). Estudios más recientes en caballos con FO de la articulación carpiana mostraron niveles significativamente incrementados de IL-6 en LS en comparación con articulaciones sanas. En cambio, el TNF no fue asociado con ese tipo de lesión articular (Ley y col 2007).

Ácido hialurónico. Durante la EA el ácido hialurónico (AH) experimenta la depolimerización de su estructura, por lo que el fluido sinovial pierde sus propiedades viscoelásticas, deteriorando la lubricación y expone al cartílago articular a una mayor fricción (Ribitsh 1990). Estudios preliminares de Hilbert y col (1984) observaron niveles de AH sinoviales significativamente bajos en caballos con artritis traumática 
de diversa consideración. Hallazgos similares reportaron Tulamo y col (1996) en caballos con distintas artropatías, en los que se observó reducción significativa del AH en LS de animales con artritis traumática crónica respecto de los controles. Del mismo modo, un estudio demostró que la concentración total y el largo de las cadenas de AH en el LS de caballos con FO fueron significativamente más bajas y cortas, respectivamente, que en los animales que no presentaron esa patología (Brown y col 2007).

\section{BIOMARCADORES DEL METABOLISMO ÓSEO}

La matriz orgánica del hueso está constituida en un 90\% por fibras de colágeno tipo I y proteínas no colágenas como la osteocalcina. El hueso es un tejido dinámico sometido a continuos procesos de síntesis y resorción. En el proceso de recambio óseo se liberan componentes de la matriz orgánica del hueso y enzimas producidas por los osteoblastos y osteoclastos, las células responsables de este recambio (Hadjidakis y Androulakis 2006). Algunos de estos elementos han sido estudiados y tienen utilidad en el monitoreo de EA en el caballo, así como en otras enfermedades del sistema musculoesquelético, como la enfermedad epifiseal, subcondral y en la predicción de fracturas (Allen 2003).

\section{BIOMARCADORES DE PROCESOS ANABÓLICOS}

Fosfatasa alcalina. La fosfatasa alcalina (FA) es una glicoproteína que sintetizan y secretan los osteoblastos, por la cual su actividad está asociada con la formación y mineralización del osteoide (Roach 1999). En caballos, la actividad de la fosfatasa alcalina específica de hueso (FAO) ha sido localizada en la superficie de los condrocitos de la placa de crecimiento, por lo que se considera como un BM de formación ósea (Henson y col 1995). Estudios de van de Lest y col (1999) en hueso subcondral equino con OC demostraron que la actividad de esta enzima se incrementa significativamente entre distintos grados de la enfermedad respecto de la actividad enzimática en el hueso subcondral normal. Este incremento de actividad FAO además podría estar correlacionado positivamente con el daño articular definido por evaluación artroscópica (Fuller y col 2001). Por otra parte, Trumble y col (2009) en un estudio en caballos de carrera con FO observaron que la actividad de la FAO en LS fue significativamente superior a los controles y diferente entre la articulación carpiana y metacarpofalángica. Además, otros autores sugieren que relacionando la actividad FAO en suero y LS tiene un valor predictivo de daño osteocondral (Trumble y col $2008^{b}$ ).

Osteocalcina. La osteocalcina $(\mathrm{Oc})$ es una proteína pequeña no colágena vitamina $\mathrm{K}$ dependiente, sintetizada principalmente por los osteoblastos y condrocitos hipertróficos del cartílago articular. La Oc se encuentra abundantemente en la MEC del hueso, y su función está asociada a la mineralización del osteoide (Gundberg 2003). Varios elementos deberían considerarse al interpretar los valores de Oc. En equinos normales los niveles séricos de Oc están determinados por distintos factores, como el sexo, ciclo circadiano, estacionalidad, ejercicio y principalmente la edad (Price 1998). Investigaciones de Frisbie y col (2003) en caballos con OC obtuvieron niveles de Oc en LS significativamente altos luego de 49 días de inducir enfermedad osteoartrítica. En cambio, las concentraciones séricas de Oc en potrillos con OC decrecieron con la edad y se correlacionaron temporalmente hasta los cinco meses de vida (Billinghurst y col 2004, Vervuert y col 2007). Asimismo, otros autores demostraron que los niveles de Oc sérica en potrillos tuvieron una fuerte correlación con el diagnóstico radiológico de OC (Donabédian y col 2008). Por último, ha sido observado un aumento significativo de la Oc sérica y sinovial en caballos osteoartríticos en ejercicio comparado con equinos normales que sólo fueron sometidos a un esfuerzo (Frisbie y col 2008).

Propéptidos de colágeno tipo I. El colágeno tipo I es el más abundante colágeno del tejido óseo. Las fracciones telopéptidas, propéptido carboxi-terminal y amino-terminal (PICP y PINP respectivamente) son extensiones liberadas del procolágeno tipo I en el proceso de maduración del colágeno tipo I. Los PICP y PINP son considerados BMs de síntesis ósea. Estudios en caballos de carrera han comprobado niveles séricos de PICP significativamente elevados asociados al ejercicio (Price y col 1995), pero sus niveles disminuyeron conforme avanzó la edad (Price y col 2001). Sin embargo, el PICP no es hueso específico, ya que el colágeno tipo I se encuentra además en otros tejidos, los cuales pueden hacer una contribución al PICP sérico (Price 1998). En efecto, un estudio encontró niveles séricos de PICP significativamente elevados en caballos con lesión del tendón flexor digital superficial, lo que sugiere que el PICP podría reflejar igualmente la síntesis de colágeno tipo I en el tejido conectivo no óseo (Jackson y col 2003).

\section{BIOMARCADORES DE PROCESOS CATABÓLICOS}

Piridinolidina-Deoxipiridinolidina. Como ocurre en los colágenos tipos II y III, las moléculas adyacentes de colágeno tipo I son estabilizadas y relacionadas por entrelazos llamados piridinolidina (Pyr) y deoxipiridinolidina (D-Pyr). Durante la degradación de la matriz ósea, los Pyr y D-Pyr son liberados a la circulación sanguínea como pequeños péptidos y luego excretados en la orina (Garnero y col 2000). Sin embargo, estos BMs tienen poca utilidad clínica diagnóstica para la EA en caballos.

Telopéptidos de colágeno tipo I. Los entrelazos telopéptidos de colágeno tipo IC-terminal (CTX-1, ICTP) y N-terminal (NTX-1) son moléculas liberadas durante la degradación del colágeno tipo I por los osteoclastos durante la resorción ósea. Los entrelazos CTX-1 y NTX-1 se liberan a 
la circulación sanguínea, siendo eliminados por la orina, pudiendo ser analizados. Sin embargo, la abundancia sérica relativa de CTX e ICTP varía de acuerdo al tipo de patología ósea, sugiriendo que estos dos fragmentos se generan en distintos sitios y vías colagenolíticas (Garnero y col 2003). Un estudio de Jackson y col (1998) sobre recambio óseo en caballos demostró que el ICTP es un BM sensible de la actividad celular ósea de recambio asociado al ejercicio, capaz de discriminar entre animales entrenados y controles sedentarios. En tanto, los niveles séricos de ICTP en caballos no demostraron ser significativamente diferentes entre ambos sexos; no obstante, existe una correlación inversa entre concentraciones séricas y la edad de los animales (Lepage y col 1998). Esto último fue confirmado posteriormente en un estudio que utilizó CTX-1 humano validado en equinos (Carstanjen y col 2004). Asimismo, en caballos con tendinitis del flexor digital superficial, el ICTP sérico no fue significativamente diferente de los valores encontrados en animales controles (Jackson y col 2003). Sin embargo, existen pruebas que el ICTP tiene un valor predictivo de enfermedades ortopédicas del desarrollo en caballos. Estudios en potrillos de carrera de dos años de edad mostraron que el valor de ICTP sérico al comenzar el programa de entrenamiento es de utilidad para identificar el riesgo de desarrollar enfermedad metacarpiana dorsal (Jackson y col 2005). En cambio, el CTX-1 sérico no pudo ser significativamente diferente entre caballos sanos y aquellos con EA interfalángica juvenil (Pastoret y col 2007).

Sialoproteína ósea. La sialoproteína ósea (SPO) es una proteína no colágena altamente específica del tejido mineralizado producida por los osteoclastos, la cual se presenta en concentraciones siete veces más altas en la interfase cartílago-hueso del hombre (Petersson y col 1998). La función de SPO en el tejido óseo no ha sido del todo aclarada, pero está relacionada con el proceso de resorción ósea, osteoclastogénesis, adhesión matriz ósea-célula y en la organización de la matriz del tejido mineralizado (Ganss y col 1999). Estudios de Ekman y col (2005) en caballos comprobaron un incremento significativo de la SPO en la interfase cartílago-hueso de áreas degenerativas del tercer hueso carpiano respecto de la interfase cartílago-hueso normal. Estos hallazgos sugieren que la SPO sería un potencial BM de cambios en el metabolismo óseo subcondral que podrían contribuir al estudio de la EA equina.

\section{BIOMARCADORES MISCELÁNEOS DE ENFERMEDAD ARTICULAR}

Mieloperoxidasa. La activación de neutrófilos en el tejido articular causa un rápido incremento de especies oxígenoreactivas y liberación de enzimas líticas como proteasas y la mieloperoxidasa (MPO) (Deby-Dupont y col 1999). Recientemente, la actividad MPO ha sido medida en diferentes tipos de EA equina. Un estudio de Fietz y col $\left(2008^{\mathrm{b}}\right)$ en
LS de caballos con OA, artritis no séptica crónica y artritis séptica demostró un aumento significativo de la actividad MPO en caballos con artritis séptica comparada con los animales controles. Este estudio sugirió que la medición de la actividad MPO en el LS podría ser útil en el estudio de la artritis séptica equina.

Proteína sensible al ácido retinoico derivada del cartílago. La proteína sensible al ácido retinoico derivada del cartílago (CD-RAP/MIA) presente fisiológicamente en el cartílago articular y patológicamente en el melanoma, es reconocida como BM de la diferenciación de los condrocitos (Bosserhoff y Buettner 2003, Andereya y col 2006). Algunos autores demostraron que las concentraciones de CD-RAP/MIA en el LS doce horas después de inducir inflamación articular fueron significativamente más altas que los valores obtenidos en los controles sanos (Berg y col 2008). Estos mismos autores sugieren que la CD-RAP/ MIA del fluido sinovial puede ser de utilidad como BM de daño articular en el caballo.

\section{COMENTARIO FINAL}

Los BMs son ampliamente usados en pacientes humanos con EA. Sin embargo, a pesar de algunas limitaciones sobre el costo y sensibilidad de las técnicas, se está estableciendo como una práctica habitual en la clínica de caballos en estos últimos años, especialmente en los equinos de deportes. Estas técnicas biomoleculares permiten obtener un diagnóstico precoz de la enfermedad articular que puede afectar a caballos atletas, evitando otros métodos diagnósticos que por su menor sensibilidad o ser invasivos pueden comprometer la progresión de la lesión articular. De este modo, este tipo de pruebas diagnósticas podrían mejorar los protocolos de tratamiento de EA, pudiendo aumentar la vida útil deportiva de los equinos. Así como han avanzado la tecnología y entendimiento de enfermedades articulares en pacientes humanos, nuestro objetivo es tener estas mismas herramientas en la clínica equina, pudiendo facilitar el diagnóstico precoz de alteraciones metabólicas del tejido articular antes que se registren imágenes de su degradación o no existan opciones en su tratamiento.

\section{RESUMEN}

La claudicación asociada a la enfermedad articular (EA) en el equino atleta es una importante causa de pérdida económica. El programa de entrenamiento intenso provoca sobrecargas biomecánicas en las articulaciones sinoviales con la activación de metaloproteinasas, lo cual ha sido relacionado con la patogénesis de la EA en el caballo, caracterizada por la degradación de la matriz extracelular del cartílago articular. Este fenómeno ocurre tempranamente en la EA provocando la liberación de componentes estructurales del cartílago al espacio articular. Así, varias moléculas componentes normales del tejido articular o subproductos del metabolismo modifican sus concentraciones en el tejido articular y líquido sinovial, por lo cual es factible medirlos y usarlos como biomarcadores moleculares de EA. Un ejemplo de esto es la evaluación en el líquido sinovial de los niveles de agrecán y de colágeno tipo II, moléculas que 
son producidas en elevada cantidad en esta patología. Este procedimiento ha permitido mejorar el entendimiento y el diagnóstico precoz de la EA en equinos. La presente revisión pretende dar una visión actualizada de los principales biomarcadores moleculares posibles de usar en EA equina, sus orígenes en el tejido articular y el uso en el estudio de la EA del caballo.

\section{REFERENCIAS}

Adarmes H, A Croxatto, M Galleguillos, E González 2006. Contenido de glicosaminoglicanos, aldehídos y proteínas en el líquido sinovial de la articulación metacarpofalángica equina normal y alterada. Arch Med Vet 38, 47-52.

Adarmes H, F Vásquez, M Galleguillos, E González. 2008. Detección de glicosaminoglicanos de la matriz del cartílago articular en el líquido sinovial de carpo equino con fractura en esquirla. Arch Med Vet 40, 77-81.

Allen MJ. 2003. Biochemical markers of bone metabolism in animals: Uses and limitations. Vet Clin Pathol 32, 101-113.

Alwan WH, SD Carter, D Bennett, SA May, GB Edwards. 1990. Cartilage breakdown in equine osteoarthritis: measurement of keratan sulphate by an ELISA system. Res Vet Sci 49, 56-60.

Alwan WH, SD Carter, D Bennett, GB Edwards. 1991. Glycosaminoglycans in horses with osteoarthritis. Equine Vet J 23, 44-47.

Andereya S, N Streich, B Schmidt-Rohlfing, T Mumme, R Müller-Rath, U Schneider. 2006. Comparison of modern marker proteins in serum and synovial fluid in patients with advanced osteoarthrosis and rheumatoid arthritis. Rheumatol Int 26, 432-438.

Arai K, K Misumi, SD Carter, S Shinbara, M Fujiki, H Sakamoto. 2005. Analysis of cartilage oligomeric matrix protein (COMP) degradation and synthesis in equine joint disease. Equine Vet J 37, 31-36.

Arai K, M Tagami, T Hatazoe, E Nishimatsu, Y Shimizu, M Fujiki, K Misumi. 2008. Analysis of cartilage oligomeric matrix protein (COMP) in synovial fluid, serum and urine, from 51 racehorses with carpal bone fracture. $J$ Vet Med Sci 70, 915-921.

Berg LC, X Mata, PD Thomsen. 2008. Molecular characterization and chromosomal assignment of equine cartilage derived retinoic acid sensitive protein (CD-Rap)/melanoma inhibitory activity (MIA). Gene 407, 98-104.

Bertone AL, LJ Palmer, L Jones. 2001. Synovial fluid cytokines and eicosanoids as markers of joint disease in horses. Vet Surg 30, 528-535.

Bertone AL, LR Bramlage, CW McIlwraith, CJ Malemud. 2005. Comparison of proteoglycan and collagen in articular cartilage of horses with naturally developing osteochondrosis and healing osteochondral fragments of experimentally induced fractures. Am $J$ Vet Res 66, 1881-1890.

Billinghurst RC, EM Buxton, MG Edwards, MS McGraw, CW McIlwraith. 2001. Use of an antineoepitope antibody for identification of typeII collagen degradation in equine articular cartilage. Am J Vet Res 62, 1031-1039.

Billinghurst RC. 2003. Biomarkers of joint disease. In: Robinson ED (ed). Current Therapy in Equine Medicine. $5^{\text {th }}$ ed. WB Saunders, USA, Pp 513-520.

Billinghurst RC, PAJ Brama, PR Van Weeren, MS Knowlton, CW McIlwraith. 2004. Evaluation of serum concentrations of biomarkers of skeletal metabolism and results of radiography as indicators of severity of osteochondrosis in foals. Am J Vet Res 65, 143-150.

Blain EJ. 2007. Mechanical regulation of matrix metalloproteinases. Front Biosci 12, 507-527.

Bolam CJ, MB Hurtig, A Cruz, BJE McEwen. 2006. Characterization of experimentally induced post-traumatic osteoarthritis in the medial femorotibial joint of horses. Am J Vet Res 67, 433-447.

Bosserhoff AK, R Buettner. 2003. Establishing the protein MIA (melanoma inhibitory activity) as a marker for chondrocyte differentiation. Biomaterials 24, 3329-3334.

Brama PA, JM Tekoppele, RA Bank, A Barneveld, PR van Weeren. 2002. Development of biochemical heterogeneity of articular cartilage: influence of age and exercise. Equine Vet J 34, 265-269.

Brama PAJ, R van den Boom, J Degroot, GH Kiers, PR van Weeren. 2004. Collagenase-1 (MMP-1) activity in equine synovial fluid
Influence of age, joint pathology, exercise and repeated arthrocentesis. Equine Vet J 36, 34-40.

Bramono DS, JC Richmond, PP Weitzel, DL Kaplan, GH Altman. 2004. Matrix metalloproteinases and their clinical applications in orthopedics. Clin Orthop Rel Res 428, 272-285.

Brown MP, TN Trumble, AH Plaas, JD Sandy, M Romano, J Hernandez, KA Merrit. 2007. Exercise and injury increase chondroitin sulfate chain length and decrease hyaluronan chain length in synovial fluid. Osteoarthr Cart 15, 1318-1325.

Carstanjen B, NR Hoyle, A Gabriel, O Hars, C Sandersen, H Amory, B Remy. 2004. Evaluation of plasma carboxy-terminal cross-linking telopeptide of Type I collagen concentration in horses. Am J Vet Res 65, 104-109.

Caterson B, CR Flannery, CE Hughes, CB Little. 2000. Mechanisms involved in cartilage proteoglycan catabolism. Matrix Biol 19, 333-344.

Céleste C, M Ionescu, AR Poole, S Laverty. 2005. Repeated intraarticular injections of triamcinolone acetonide alter cartilage matrix metabolism measured by biomarkers in synovial fluid. J Orthop Res 23, 602-610.

Clegg PD, RM Burke, AR Coughlan, CM Riggs, SD Carter. 1997ª Characterization of equine matrix metalloproteinase 2 and 9; and identification of the cellular sources of these enzymes in joints. Equine Vet J 29, 335-342.

Clegg PD, AR Coughlan, CM Riggs, SD Carter. 1997b. Matrix metalloproteinases 2 and 9 in equine synovial fluids. Equine Vet J 29, 343-348.

Clegg PD, SD Carter. 1999. Matrix metalloproteinase -2 and -9 are activated in joint disease. Equine Vet J 31, 324-330.

Clutterbuck AL, P Harris, D Allaway, A Mobasheri. 2008. Matrix metalloproteinases in inflammatory pathologies of the horses. Vet Journal. In press.

Cruz AM, MB Hurting. 2008. Multiple pathways to osteoarthritis and articular fractures: is subchondral bone the culprit? Vet Clin North Am Equine Prac 24, 101-116.

D'Lima DD, S Hashimoto, PC Chen, CW Colwell, MK Lotz. 2001 Cartilage injury. Impact of mechanical trauma on matrix and cells. Clin Orthop Rel Res 391S, 90-99.

de Grauw JC, CH van de Lest, R van Weeren, H Brommer, PA Brama. $2006^{\mathrm{a}}$. Arthrogenic lameness of the fetlock: synovial fluid markers of inflammation and cartilage turnover in relation to clinical joint pain. Equine Vet J 38, 305-311

de Grauw JC, PA Brama, P Wiemer, $\mathrm{H}$ Brommer, $\mathrm{CH}$ van de Lest, PR Weeren. 2006 ${ }^{\mathrm{b}}$. Cartilage-derived biomarkers and lipid mediators of inflammation in horses with osteochondritis dissecans of the distal intermediate ridge of the tibia. Am J Vet Res 67, 1156-1162.

Deby-Dupont G, C Deby, M Lamy. 1999 Neutrophil myeloperoxidase revisited: It's role in health and disease. Intensivmed 36, 500-513.

Dijkgraaf LC, LMG DeBont, G Boering, RS Liem.1995. Normal cartilage structure, biochemistry, and metabolism: A Review of the literature. J Oral Maxillofac Surg 53, 925-929.

Donabédian M, PR van Weeren, G Perona, G Fleurance, C Robert, S Léger, D Bergero, O Lepage, W Martin-Rosset. 2008. Early changes in biomarkers of skeletal metabolism and their association to the occurrence of osteochondrosis (OC) in the horse. Equine Vet J 40, 253-259.

Ekman S, E Skiöldebrand, D Heinegarg, K Hultenby. 2005. Ultrastructural immunolocalisation of bones sialoprotein in the osteocartilagenous interface of the equine third carpal bone. Equine Vet J 37, 26-30.

Eyre D. 2002. Collagen of articular cartilage. Arthritis Res 4, 30-35.

Fietz S, R Einspanier, S Hoppner, B Hertsch, A Bondzio. 2008 . Determination of MMP-2 and MMP-9 activities in synovial fluid of horses with osteoarthritic and arthritic joint disease using gelating zymography and immunocapture activity assays. Equine Vet $J$ 40, 266-271.

Fietz S, A Bondzio, A Moschos, B Hertsch, R Einspanier. 2008 ${ }^{\mathrm{b}}$ Measurement of equine myeloperoxidase (MPO) activity in synovial fluid by a modified MPO assay and evaluation of joint disease- an initial case study. Res Vet Sci 84, 347-353.

Fortier LA, MA Kormatowsaima, HO Mohammed, MT Jordan, LC Cain, WB Steven. 2005. Age-related changes in serum insulin-like growth factor-1, binding protein-3, and articular cartilage structure in Thoroughbred horses. Equine Vet J 37, 37-42. 
Fradette ME, C Céleste, H Richard, G Beauchamp, S Laverty. 2007. Effects of continuous oral administration of phenylbutazone on biomarkers of cartilage and bone metabolism in horses. Am J Vet Res $68,128-133$.

Frisbie DD, CS Ray, M Ionescu, AR Poole, PL Chapman, CW Mcllwraith 1999. Measurement of synovial fluid and serum concentrations of the 846 epitope of chondroitin sulfate and of carboxy propeptides of type 11 procollagen for diagnosis of osteochondral fragmentation in horses. Am J Vet Res 60, 306-309.

Frisbie DD. 2002. Markers of osteoarthritis: Implications for early diagnosis and monitoring of pathological course and effects of therapy. In: Ross MW, Dyson SJ (eds). Diagnosis and Management of Lameness in the Horse. WB Saunders. USA, Pp 591-594.

Frisbie DD, FA Al-Sobayil, RC Billinghurst, CW McIlwraith. 2003. Synovial fluid biomarkers distinguish exercise from osteoarthritis pathology. AAEP Proceedings 49, 116-117.

Frisbie DD, E Duffy, R Arthur, J Blea, V Baker, RC Billinghurst, CW McIlwraith. 2005. Prospective clinical study assessing serum biomarkers for musculoskeletal disease in 2-to 3-yr-old racing Thoroughbreds. AAEP Proceeding 51.

Frisbie DD, FAl-Sobayil, RC Billinghurst, CE Kawcak, CW McIlwraith 2008. Changes in synovial fluid and serum biomarkers with exercise and early osteoarthritis in horses. Osteoarthr Cartilage 16, 1196-1204.

Fuller CJ, ARS Barr, PA Dieppe, M Sharif. 1996. Variation of an epitope of keratan sulphate and total glycosaminoglycans in normal equine joints. Equine Vet J 28, 490-493.

Fuller CJ, ARS Barr, M Sharif, PA Dieppe. 2001. Cross-sectional comparison of synovial fluid biochemical markers in equine osteoarthritis and the correlation of these markers with articular cartilage damage. Osteoarthr Cartilage 9, 49-55.

Ganss B, RH Kim, J Sodek. 1999. Bone sialoprotein. Crit Rev Oral Biol Med 10, 79-98.

Garnero P, JC Rousseau, PD Delmas. 2000. Molecular basis and clinica use of biochemical markers of bone, cartilage, and synovium in joint diseases. Arthr Rheum 43, 953- 968.

Garnero P, M Ferreras, MA Karsdal, R Nicamhlaoibh, J Ristell, O Borel, P Qvist, PD Delmas, NT Foged, JM Delaisse. 2003. The type I collagen fragments ICTP and CTX reveal distinct enzymatic pathways of bone collagen degradation. J Bone Min Res 18, 859-867.

Gibson KT, H Hodge, T Whittem. 1996. Inflammatory mediators in equine synovial fluid. Aust Vet $J$ 73, 148 -151.

Goldring SR, MB Goldring. 2004. The rol of cytokines in cartilage matrix degeneration in osteoarthritis. Clin Orthop Rel Res 427S, 27-36.

Gundberg CM. 2003. Matrix proteins. Osteoporos Int 14S, 37-42.

Hadjidakis DJ, II Androulakis. 2006. Bone remodeling. Ann N Y Acad Sci 1092, 385-396.

Hall AC, PG Bush, MEB Davidson, SA Kempson. 2003. Equine articular cartilage chondrocytes: Opening the black box. Equine Vet $J 35,425-428$

Hardingham T, AJ Fosang. 1995. The structure of aggrecan and its turnover in cartilage. J Rheumatol Suppl 43, 86-90.

Helal IE, K Misumi, O Tateno, T Kodama, M Ishimaru, J Yamamoto, D Miyakoshi, M Fujiki. 2007. Effect of exercise on serum concentration of cartilage oligomeric matrix protein in Thoroughbreds. Am J Vet Res 68, 134-140.

Henson FMD, ME Davies, JN Skepper, L Jeffcott. 1995. Localization of alkaline phosphatase in equine growth cartilage. J Anat 187 , 151-159.

Hilbert BJ, G Rowley, KN Antonas. 1984. Hyaluronic acid concentration in synovial fluid from normal and arthritic joint of horses. Aust Vet $J 61,22-24$

Hozokawa R, Y Uchida, S Fujiwara, T Noguchi. 1988. Lactate dehydrogenase isoenzymes are present in matrix vesicles. $J$ Biol Chem 263, 10045-10047.

Huber M, S Trattnig, F Lintner. 2000. Anatomy, biochemistry, and physiology of articular cartilage. Invest Radiol 35, 573-580.

Hultén C, U Gronlund, J Hirvonen, RM Tulamo, MM Suominen, G Marhaug, M Forsberg. 2002. Dynamics in serum of the inflammatory markers serum amyloid A (SAA), haptaglobin, fibrinogen and $\alpha 2$-globulins during induced noninfectious arthritis in the horse. Equine Vet $J$ $34,699-704$.
Jackson BF, R Eastell, AM Wilson, LE Lanyon, AG Gooship, JS Price. 1998. The effect of exercise on biochemical markers of bone turnover and insulin-like growth factor I in two year old Thoroughbreds. J Bone Miner Res 13, 521.

Jackson BF, RKW Smith, JS Price. 2003. A molecular marker of typo I collagen metabolism reflects changes in connective tissue remodeling associated with injury to the equine superficial digital flexor tendon. Equine Vet J 35, 211-213.

Jackson BF, C Lonell, KL Verheyen, P Dyson, DU Pfeiffer, JS Price. 2005. Biochemical markers of bone metabolism and risk of dorsal metacarpal disease in 2-year-old Thoroughbreds. Equine Vet J 37, 87-91.

Jacobsen S, MH Thomsen, S Nanni. 2006. Concentrations of serum amyloid A in serum and synovial fluid from healthy horses and horses with joint disease. Am J Vet Res 67, 1738-1742.

Jansson N. 1996. Equine osteoarthritis: A review of pathogenesis, diagnosis and treatment. Pferdeheilkunde 12, 111-118.

Karopoulos C, MJ Rowley, MZ Ilic, CJ Handley. 1996. Presence of antibodies to native $\mathrm{G} 1$ domain of aggrecan core protein in synovial fluids from patients with various joint diseases. Arthritis Rheum 39, 1990-1997.

Kawcak CE, CW McIlwraith, RW Norrdin, RD Park, SP James. 2001. The role of subchondral bone in joint disease: A review. Equine Vet J 33, 120-126.

Kidd JA, C Fuller, ARS Barr. 2001. Osteoarthritis in the horses. Equine Vet Educ 13,160-168.

Kidd JA, AR Barr, JF Tarlton. 2007. Use of metalloproteinases 2 and 9 and white blood cell counts in monitoring the treatment and predicting the survival of horses with septic arthritis. Vet Rec 161, 329-334.

Kim YI, RL Sah, AJ Grodzinsky, AH Plaas, JD Sandy. 1994. Mechanical regulation of cartilage biosynthetic behavior: physical stimuli. Arch Biochem Biophys 311, 1-12.

Laverty S, M Ionescu, M Marcoux, L Bouré, B Doizé, AR Poole. 2000. Alterations in cartilage type II procollagen and aggrecan contents in synovial fluid in equine osteochondrosis. J Orthop Res 18 , 339-405.

Lepage OM, DJ Hartmann, R Eicher, B Uebelhart, P Tschudi, D Uebelhart. 1998. Biochemical markers of bone metabolism in draught and warmblood horses. Vet Journal 156, 169-175.

Lepage OM, B Carstenjen, D Uebelhart. 2001. Non-invasive assessment of equine bone: An update. Vet J 161, 10-22.

Ley C, S Ekman, A Elmén, G Nilsson, ML Eloranta. 2007. Interleukin-6 and tumour necrosis factor in synovial fluid from horses with carpal joint pathology. J Vet Med A Physiol Pathol Clin Med 54, 346-351.

Mathews C, KE Vanholde, KG Ahern. 2002. Bioquímica. $3^{\text {a }}$ ed. Addison Wesley Iberoamericana España, Madrid, España.

McIlwraith CW. 1996. General pathobiology of the joint and response to injury. En: McIlwraith CW, Trotter GW. Joint Disease in the Horse. WB Saunders (eds). USA, Pp 40-70.

McIlwraith CW, RC Billinghurst, DD Frisbie. 2001. Current and future diagnostic means to better characterize osteoarthritis in the horseRoutine synovial fluid analysis and synovial fluid and serum markers. AAEP Proceedings 47, 171-179.

McIlwraith CW. 2002. Arthroscopic surgery for osteochondral chip fragments and other lesions not requiring internal fixation in the carpal and fetlock of the equine athlete: What have we learned in 20 years? Clin Tech Equine Prac 1, 200-210.

McIlwraith CW. 2005. Use of synovial fluid and serum biomarker in equine bone and joint disease: A review. Equine Vet J 37, 473-482.

Misumi K, V Vilim, T Hatazoe, T Murata, M Fujiki, T Oka, H Sakamoto, SD Carter. 2002. Serum level of cartilage oligomeric matrix protein (COMP) in equine osteoarthritis. Equine Vet $J 34,602-608$.

Misumi K, M Tagami, T Kamimura, D Miyakoshi, IE Helal, K Arai, M Fujiki. 2006. Urine cartilage matrix protein (COMP) measurement is used in discriminating the osteoarthritis Thoroughbreds. Osteoarthr Cart 14, 1174-1180.

Muller-Gerbl M.1998. The subchondral bone plate. Ad. Anat. Emb Cell Biol 141, 1-134.

Oke SL, MB Hurtig, RA Keates, JR Wright, JH Lumsden. 2003. Assessment of three variations of the 1,9-dimethylmethylene blue assay for measurement of sulfated glycosaminoglycan concentrations in equine synovial fluid. Am J Vet Res 64, 900-906. 
Okumura M, GH Kim, M Tagami, S Haramaki, T Fujinaga. 2002. Serum keratan sulphate as a cartilage metabolic marker in horses: The effect of exercise. J Vet Med Physiol Clin Med 49, 195-197.

Palmer JL, AL Bertone. 1994. Joint structure, biochemistry and biochemical disequilibrium in synovitis and equine joint disease. Equine Vet J 26, 263-277.

Park RD, PF Steyn, RH Wrigley. 1996. Imaging techniques in the diagnosis of equine joint disease. In: CW McIlwraith, GW Trotter (eds). Joint Disease in the Horse. Philadelphia, WB Saunders, USA, Pp 145-164.

Pastoret V, B Carstanjen, JP Lejeune, F Karnir, B Remy, JY Reginster, D Serteyn, A Gabriel. 2007. Evaluation of serum osteocalcin and CTX-1 in Ardenner horses with special reference to juvenile interphalangeal joint disease. J Vet Med A Physiol Pathol Clin Med 54, 458-463.

Petersson IF, T Boegard, J Dahlstrom, B Svensson, D Heinegard, T Saxne. 1998. Bone scan and serum markers of bone and cartilage in patients with knee pain and osteoarthritis. Osteoarthr Cartilage 6, 33-39.

Platt D, JLE Bird, MT Bayliss. 1998. Ageing of equine articular cartilage: Structure and composition of aggrecan and decorin. Equine Vet $J$ 30, 43-52.

Poole AR. 1993. Cartilage in health and disease. In: McCarty DJ (ed). Arthritis and Allied Conditions. A Texbook of Rheumatology. Lea \& Febiger, Philadelphia, USA, Pp 279-333.

Poole AR, T Kojima, T Vasuda, F Mwale, M Kobayashi, S Laverty. 2001. Cartilage biology. Composition and structure of articular cartilage. Clin Orthop Rel Res 391S, 26-33.

Preston SA, TN Trumble, DN Zimmel, TL Chmielewski, MP Brown, JA Hernández. 2008. Lameness, athletic performance, and financial returns in yearling Thoroughbreds bought for the purpose of resale for profit. J Am Vet Med Assoc 232, 85-90.

Price JS, B Jackson, R Eastell, AM Wilson, RCJ Rusell, LE Lanyon, AE Goodship. 1995. The response of the skeleton to physical training: A biochemical study in horses. Bone 17, 221-229.

Price JS.1998. Biochemical markers of bone metabolism in horses: Potentials and limitations? Vet Journal 156, 163-165.

Price JB, BF Jackon, JA Gray, PA Harris, IM Wright, DU Pfeiffer, SP Robins, R Eastell, SW Ricketts. 2001. Biochemical markers of bone metabolism in growing Thoroughbred: A longitudinal study. Res Vet Sci 71, 37-44.

Ray CS, AR Poole, CW Mcllwraith. 1996. Use of synovial fluid and serum markers in articular disease. In: McIwraith CW, Trotter GW (eds). Joint Disease in the Horse. WB Saunders, Philadelphia, USA, Pp 203-216.

Ribitsh VO. 1990. Viscostatic behavior of synovial fluids and artficial replacements. In: Mow VC, Woo SLY, Ratcliffe A (eds). Biomechanics of Diarthrodial Joints. Vol II. Springer-Verlag. New York, USA, Pp 287-304.

Rizkalla G, A Reiner, E Bogoch, AR Poole. 1992. Studies of the articular proteoglycan aggrecan in health and osteoarthritis. $J$ Clin Invest 90, 2268-2277.

Roach H. 1999. Association of matrix acid and alkaline phosphatases with mineralization of cartilage and endochondral bone. Histochem $J 31,53-61$.

Robion FC, B Doizé, L Bouré, M Marcoux, M Ionescu, A Reiner, AR Poole, S Laverty. 2001. Use of sinovial fluid markers of cartilage synthesis and turnover to study effects of repeated intra-articular administration of methylprednisolone acetate on articular cartilage in vivo. J Orthop Res 19, 250-258.

Rosenberg L. 1993. Structure and function of cartilage proteoglycans. In: McCarty DJ (ed). Arthritis and Allied Conditions. A Texbook of Rheumatology. Lea \& Febiger, Philadelphia, USA, Pp 229-243.

Rosenberg K, H Olsson, M Mórgelin, D Heinegard. 1998. Cartilage oligomeric matrix protein shows high affinity $\mathrm{Zn}$-dependent interaction with triple helical collagen. J Biol Chem 273, 20397-20403.

Roughley PJ. 2006. The structure and function of cartilage proteoglycans. Eur Cell Mater 12, 92-101.
Semevolos SA, AJ Nixon, BD Brower-Toland. 2001. Changes in molecular expression of aggrecan and collagen types I, II and X, insulin-like growth factor-l, and transforming growth factor- $\beta$ in articular cartilage obtained from horses with naturally acquired osteochondrosis. Am J Vet Res 62, 1088- 1094.

Skiöldebrand E, P Lorenzo, L Zunino, GJ Rucklidge, B Sandgren, J Carlsten, S Ekman. 2001. Concentration of collagen, aggrecan and cartilage oligomeric matrix protein (COMP) in synovial fluid from equine middle carpal joints. Equine Vet J 33, 394-402.

Skiöldebrand E, D Heinegard, ML Eloranta, G Nilsson, J Dudhia, B Sandgren, S Ekman. 2005. Enhanced concentration of COMP (cartilage oligomeric matrix protein) in osteochondral fractures from racing Thoroughbreds. J Orthop Res 23,156-163.

Smith RKW, D Heinegard. 2000. Cartilage oligomeric matrix protein (COMP) levels in digital sheath synovial and serum with tendon injury. Equine Vet $J 32,52-58$.

Stell CM. 2008. Equine synovial fluid analysis. Vet Clin North Am Equine Pract 24, 437-454.

Sutton S, A Clutterbuck, P Harris, T Gent, S Freeman, N Foster, R Barret-Jolley, A Mobasheri. 2009. The contribution of the synovium, synovial derived inflammatory cytokines and neuropeptides to the pathogenesis of osteoarthritis. Vet $J 179,10-24$.

Todhunter RJ, AE Yeager, KP Freeman, EJ Parente, G Lust. 1993. Keratan sulfate as a marker of articular cartilage catabolism and joint treatment in ponies. Am J Vet Res 54, 1007-1016.

Todhunter RJ. 1996. Anatomy and physiology of synovial joints. In McIllwraith JW, Trotter GW (eds). Joint Disease in the Horse. WB Saunders, USA, Pp 1-28.

Trumble TN, GW Trotter, JRT Oxford, CW McIlwraith, S Cammarata, JL Goodnight, RC Billinghurst, DD Frisbie. 2001. Synovial fluid gelatinase concentrations and matrix metalloproteinase and cytokine expression in naturally occurring joint disease in horse. Am J Vet Res 62, 1467-1477.

Trumble TN, AB Scarbrough, MP Brown. 2009. Osteochondral injury increases type II collagen products $(\mathrm{C} 2 \mathrm{C})$ in synovial fluid of Thoroughbred racehorses. Osteoarthr Cartilage 17, 371-374.

Trumbe TN, MP Brown, KA Merrit, RC, Billinghurst. 2008b. Joint dependent concentration of bone alkaline phosphatase in serum and synovial fluids of horses with osteochondral injury: an analytical and clinical validation. Osteoarthr Cartilage 16, 779-786.

Tulamo R-M, J Houttu, A Tupamaki, M Salonen. 1996. Hyaluronate and large molecular weight proteoglycans in synovial fluid from horses with various arthritides. Am J Vet Res 57, 932-937.

Vachon AM, FW Keely, CW McIlwraith, P Chapman. 1990. Biochemical analysis of normal articular cartilage in horses. Am JVet Res 51, 1905-1911.

van de Lest $\mathrm{CH}$, BM van den Hoogen, PR van Weeren, JF Browers, LM van Golde, A Barneveld. 1999. Changes in bone morphogenic enzymes and lipid composition of equine osteochondrotic subchondral bone. Equine Vet $J$ (S) 31, 31-37.

van den Boom R, MR van der Harst, H Brommer, PAJ Brama, A Barneveld, PR van Weeren, J DeGroot. 2005. Relationship between synovial fluid levels of glycosaminoglycans, hydroxyproline, and general MMP activity and the presence and severity of articular change in the proximal articular surface of P1. Equine Vet J 37, 19-25.

van Weeren PR, EC Firth. 2008. Future tools for early diagnosis and monitoring of musculoskeletal injury: biomarkers and CT. Vet Clin North Am Equine Pract 24, 153-175.

Vervuert I, S Winkelsett, L Christmann, E Bruns, HO Hoppen, O Distl, B Hertsch, M Coenen. 2007. Evaluation of the influences of exercise, birth date, and osteochondrosis on plasma bone marker concentrations in Hanoverian Warmblood foals. Am J Vet Res 68, 1319-1323.

von Rechenberg B, C Leutenegger, K Zlinsky, CW McIlwraith, MK Akens, JA Auer. 2001. Upregulation of mRNA of interleukin -1 and -6 in subchondral cystic lesions of four horses. Equine Vet J 33, 143-149.

Yancik SA, CW McIlwraith, AE Wagner, GW Trotter. 1987. Evaluation of creatine kinase and lactate dehydrogenase activities in clinically normal and abnormal equine joints. Am J Vet Res 48, 463-466. 